Ann. Biol. anim. Bioch. Biophys., I974, 14 (3), 4II-4I5.

\title{
MÉTHODE DE SUPPRESSION RÉVERSIBLE DE L'ODORAT CHEZ LA BREBIS ET VÉRIFICATION DE L'ANOSMIE AU MOYEN D'UNE ÉPREUVE COMPORTEMENTALE
}

\author{
P. POINDRON \\ avec la collaboration technique de Y. Lebreton, P. Orgeur et Colette Lavenet
}

\begin{abstract}
Station de Physiologie de la Reproduction, Centre de Recherches de Tours, I. N. R. A., Nouzilly B. P. 1, 37380 Monnaie
\end{abstract}

\section{RÉSUMÉ}

Une anosmie a été induite chez la Brebis par irrigation des fosses nasales avec une solution de sulfate de zinc à I p. roo additionnée de Procaïne à 3 p. Ioo. L'acceptation ou le refus de nourriture associée à une odeur répulsive a permis de connaitre la durée de l'anosmie ainsi induite. Dix brebis âgées de deux ans ont été utilisées. Quatre heures après le traitement aucun des dix animaux n'a refusé la nourriture. La durée de l'anosmie a été variable, allant de moins de huit heures pour trois animaux à plus de vingt-cinq jours pour trois autres.

Chez la Brebis, l'anosmie est généralement obtenue par ablation des bulbes olfactifs (Bouissou, I968; Morgan, Arnold et Lindsay, 1972). Cette technique présente plusieurs inconvénients qui limitent son emploi pour aborder des études faisant intervenir la sensibilité olfactive : d'une part les lésions nerveuses occasionnées ainsi que le traumatisme opératoire peuvent interférer avec les effets de l'anosmie; d'autre part le caractère irréversible de ce type d'intervention interdit de nombreux schémas d'expérimentation.

Une anosmie temporaire est obtenue chez les Rongeurs par action d'une solution de sulfate de zinc sur la muqueuse olfactive (ALBERTS et GALEF, I97I). Nous avons adapté cette méthode aux Ovins. Pour en vérifier l'efficacité une épreuve de discrimination olfactive a d'abord été mise au point. 


\section{I. - ÉPREUVE DE DISCRIMINATION OLFAC'TIVE}

Certaines sécrétions odorantes produites par une espèce peuvent avoir un effet répulisif sur une autre espèce. C'est le cas pour l'urine de Renard vis-à-vis du Rat (Vernet-Maury, I968). D'après une tradition de bergers les excréments de Chien sont réputés pour leur valeur répulsive vis-à-vis des Ovins. Nous avons pu vérifier qu'effectivement des brebis refusent toute nourriture imprégnée de l'odeur d'excrément de chien.

\section{Déroulement des épreuves}

Deux bacs métalliques identiques sont disposés dans une salle. Leur position respective ainsi que leur situation dans la pièce sont modifiées au hasard pour chaque série d'épreuves. Chaque récipient comprend une grille à trame très fine, sous laquelle se trouve un espace libre. Dans l'un des deux bacs, de l'excrément frais de Chien est placé sous la grille; dans l'autre bac l'espace est laissé vide. De l'avoine additionnée de maîs déshydraté est alors disposée sur la grille de chaque récipient. Ainsi l'aliment n'est pas en contact avec la matière répulsive. Les animaux, à jeun depuis 24 heures, sont introduits dans la pièce, deux par deux pour éviter les réactions de peur, très générales chez les brebis isolées.

Les critères utilisés au cours d'une épreuve sont définis ci-dessous. Nous considérons que :

- une brebis qui mange plus de cinq secondes dans le bac contenant l'excrément ne présente pas de discrimination olfactive;

- une brebis qui mange dans le bac sans odeur répulsive, et qui mange moins de cinq secondes dans l'autre après l'avoir flairé, présente une discrimination olfactive ;

- dans le cas où un animal mange dans le bac ne contenant pas d'excrément et ne flaire pas l'autre, l'épreuve n'est pas retenue. Il en est de même lorsqu'une brebis ne mange dans aucun des deux bacs.

\section{Valeur répulsive de l'excrément de chien, résultats}

Dix brebis de race Ile-de-France âgées de 2 ans, ainsi que 2 brebis de race Préalpes $d u$ Sud âgées de 5 ans, ont été utilisées. Les I 2 animaux ont été testés cinq fois chacun à raison d'une fois par jour. Au cours des soixante épreuves ainsi réalisées, aucune brebis n'a mangé dans le récipient contenant la matière répulsive (tabl. I) (quelques essais réalisés sur des Bovins et des Caprins semblent indiquer que la valeur répulsive de l'excrément de Chien est également très marquée pour ces deux espèces).

\section{II. - TECHNIQUE DE SUPPRESSION}

\section{DE LA DISCRIMINATION OLFACTIVE}

Des essais préliminaires nous ont montré que l'utilisation sans modification de la méthode d'ALBERTS et GALEF (irrigation de la muqueuse nasale par une solution de sulfate de Zinc à 5 p. IOo) provoque un état de choc extrême et une mortalité élevée chez nos animaux. 
SUPPRESSION DE L'ODORAT CHEZ I,A BREBIS

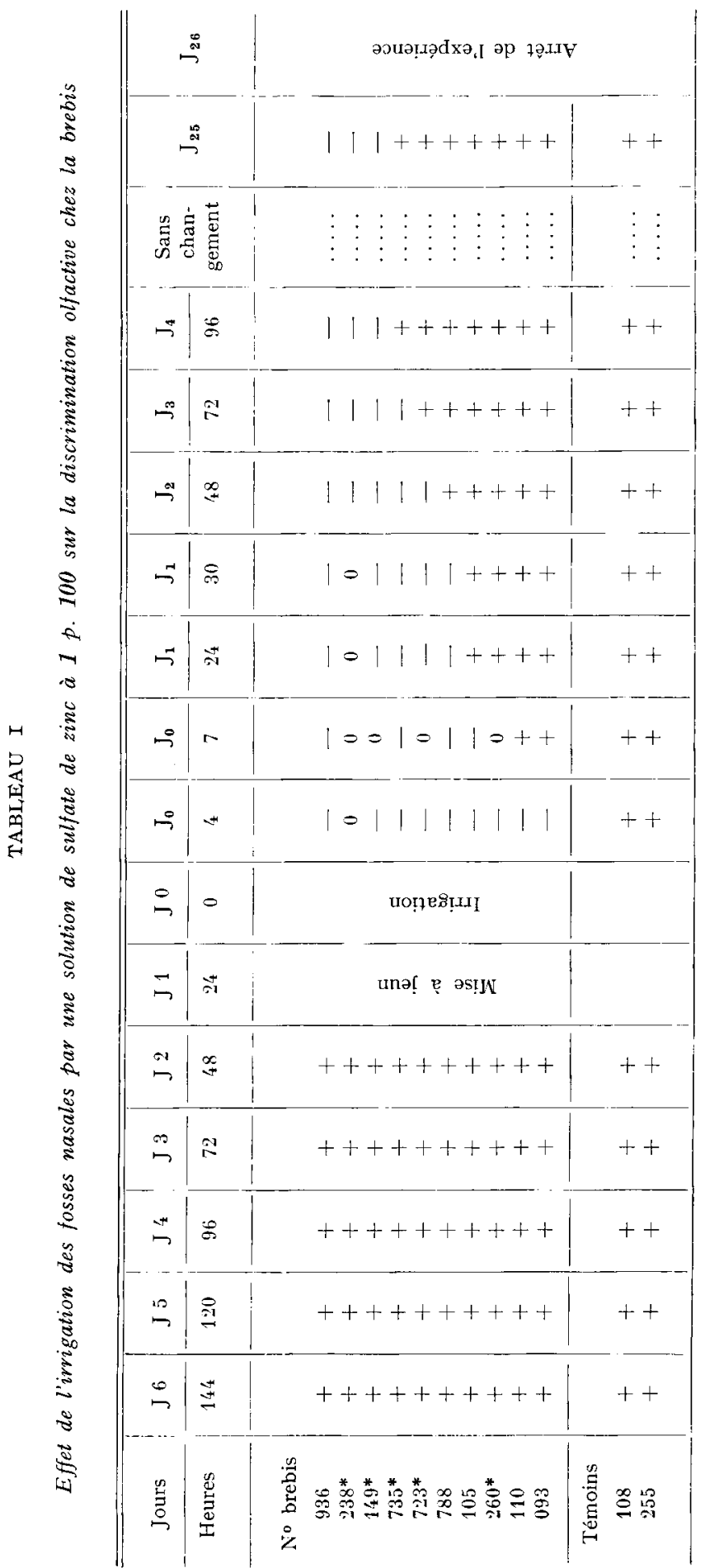


Nous avons donc été amenés à réduire la concentration de sulfate de Zinc. D'autre part, un arrêt de la respiration se produit fréquemment au moment de l'introduction de la solution dans les fosses nasales. Ce phénomène ne s'est pas reproduit après adjonction de chlorydrate de procaïne à la solution.

\section{Technique d'irrigation}

Les dix animaux Ile-de-France ont subi le traitement, tandis que les deux brebis Préalpes ont été conservées intactes pour vérifier journellement la valeur répulsive de l'excrément. Chaque animal subit une injection de $3 \mathrm{ml}$ d'acétate de cortisone à $25 \mathrm{mg} / \mathrm{ml}$ trois heures avant l'intervention et $2 \mathrm{ml}$ de phénergan à $25 \mathrm{mg} / \mathrm{ml}$ au moment de l'irrigation, ceci afin de combattre un éventuel état de choc consécutif à la destruction de la muqueuse nasale.

Les brebis sont anesthésiées par inhalation de fluothane. Une intubation trachéale est réalisée afin d'éviter la pénétration du liquide dans les voies respiratoires.

L'animal est ensuite placé sur le dos. Une solution de sulfate de Zinc à I p. Ioo additionnée de chlorydrate de procaïne à $3 \mathrm{p}$. Ioo est introduite par les narines, à l'aide d'une seringue prolongée par un fin cathéter. L'irrigation est interrompue lorsque la solution ressort de chaque narine. Les volumes de liquide introduits ont varié de 80 à $\mathrm{r} 30 \mathrm{ml}$ par brebis. L'animal est ensuite maintenu 3 minutes puis placé la tête en bas, de manière à favoriser l'écoulement de la solution. Cinq brebis sur dix ont ensuite subi un rinçage des fosses nasales à raison de $30 \mathrm{ml}$ de sérum physiologique par narine.

\section{RÉSULTATS}

L'effet de l'irrigation sur les performances olfactives de nos animaux est présenté dans le tableau $\mathbf{I}$.

L'effet du traitement est très marqué puisque quatre heures après 1'irrigation, aucun des animaux traité n'a manifesté de discrimination olfactive entre les deux récipients.

Par contre la durée de l'anosmie ainsi induite est très variable puisqu'elle peut aller de quatre heures à vingt-cinq jours.

Enfin le fait de procéder à un rinçage des fosses nasales ne modifie pas l'efficacité du traitement de façon significative.

Nous disposons donc d'une technique de suppression réversible de l'odorat chez les Ovins, ainsi que d'une méthode simple et efficace pour contrôler la capacité de discrimination olfactive de ces animaux. Ceci permet d'envisager de nombreuses études expérimentales, étant donné l'importance que semblent revêtir les informations olfactives dans l'organisation des comportements social, sexuel et maternel des Ongulés. 


\section{REMERCIEMENTS}

Je désire remercier tout particulièrement MM. Petrequin et P. Richard qui nous ont indiqué les propriétés répulsives de l'excrément de Chien. Je tiens également à remercier MM. J. P. Signoret et P. MacLEod, ainsi que Mile M. F. Bourssou pour leurs remarques et suggestions à propos de ce travail. Enfin je désire remercier M. M. Dubors qui nous a fait profiter de ses connaissances vétérinaires.

\section{SUMMARY}

\section{INDUCTION OF ANOSMIA IN THE EWE AND BEHAVIORAL TEST OF THE OLFACTORY DEFICIT}

Anosmia was induced in the ewe by irrigation of the nasal mucosa with a I p. Ioo solution of zinc sulphate with 3 p. I 00 Procaine added. A behavioral test was developed to assess olfactory deficit; this consisted in the acceptance or refusal of food associated with a repulsive odor. Ten two-year old ewes were treated. Four hours after the treatment, all the animals were unable to react to the repulsive odor. The duration of anosmia is highly variable, ranging from less than eight hours to more than twenty-five days.

\section{RÉFÉRENCES BIBLIOGRAPHIQUES}

Alberts J. R., Galef B. G., I97I. Acute anosmia in the Rat : a behavioral test of a peripherallyinduced olfactory deficit. Physiol. Behav., 6, 619-621.

Bourssou M. F., I968. Effet de l'ablation des bulbes olfactifs sur la reconnaissance du jeune par sa mere chez les Ovins. Rev. Comp. Animal, 3, 77-83.

Morgan P. D., Arnold G. W., Lindsay D. R., I972. A note on the mating behaviour of ewes with various senses impaired. J. Reprod. Fert., 30, I5 I-I52.

Vernet-Maury E., Le Magnen J., Chanel J., Ig68. Comportement émotif chez le rat; influence de l'odeur d'un prédateur et d'un non-prédateur. C. R. Acad. Sci., 267, 33 I-334. 